\title{
On the connection problem for nonlinear differential equation
}

\section{Zhao-Yun Zeng ${ }^{1 *}$ (D) and Lin Hu'}

"Correspondence: jenseng5@163.com

'School of Mathematics and Physics, Jinggangshan University, Ji'an, China

\section{Abstract}

We consider the connection problem of the second nonlinear differential equation

$$
\Phi^{\prime \prime}(x)=\left(\Phi^{\prime 2}(x)-1\right) \cot \Phi(x)+\frac{1}{x}\left(1-\Phi^{\prime}(x)\right)
$$

subject to the boundary condition $\Phi(x)=x-a x^{2}+O\left(x^{3}\right)$ as $x \rightarrow 0$. In view of the fact that equation ( 1 ) is equivalent to the fifth Painlevé (PV) equation after a Möbius transformation, we are able to study the connection problem of equation (1) by investigating the corresponding connection problem of PV. Our research technique is based on the method of uniform asymptotics presented by Bassom et al. The asymptotic behavior of the monotonic solution as $x \rightarrow \infty$ on the real axis of equation (1) is obtained, the explicit relation (connection formula) between the constants appearing in the asymptotic behavior and the real number $a$ are also obtained.

Keywords: Connection formulas; Uniform asymptotics; Painlevé V equation; Parabolic cylinder function

\section{Introduction and main results}

In the present paper we show how the technique of uniform asymptotics introduced by Bassom et al. in [1] can be applied to the equation

$$
\Phi^{\prime \prime}(x)=\left(\Phi^{\prime 2}(x)-1\right) \cot \Phi(x)+\frac{1}{x}\left(1-\Phi^{\prime}(x)\right)
$$

whose solutions are related to the computation of one particle density matrix of impenetrable bosons at zero temperature $[4,16]$.

We focus on the problem of calculating the asymptotic behaviors as $x \rightarrow \infty$ of a oneparameter family of regular solutions to equation (2) defined with the boundary condition

$$
\Phi(x)=x-a x^{2}+O\left(x^{3}\right), \quad \text { as } x \rightarrow 0,
$$

and on the relevant connection formulas between the different asymptotic parameters which appeared in the above-mentioned critical expansions.

Introducing the change of variable

$$
y(s)=\exp (-2 i \Phi(x)), \quad s=\frac{x}{2}
$$

(c) The Author(s) 2019. This article is distributed under the terms of the Creative Commons Attribution 4.0 International License (http://creativecommons.org/licenses/by/4.0/), which permits unrestricted use, distribution, and reproduction in any medium, provided you give appropriate credit to the original author(s) and the source, provide a link to the Creative Commons license, and indicate if changes were made. 
in (2), we get for $y(s)$ the special fifth Painlevé equation (PV)

$$
\frac{d^{2} y}{d s^{2}}=\left(\frac{1}{2 y}+\frac{1}{y-1}\right)\left(\frac{d y}{d s}\right)^{2}-\frac{1}{s} \frac{d y}{d s}-4 i \frac{y}{s}+8 \frac{y(y+1)}{y-1}
$$

which appears in the studying of the level spacing functions related to the Fredholm determinant of the sine kernel $\frac{\sin \pi(x-y)}{\pi(x-y)}$ on the finite interval $(-s, s)[11,14]$. Let $s=i t$ and $p(t)=\frac{\sqrt{y}+1}{\sqrt{y}-1}$, then equation (5) is equivalent to a special third Painlevé (PIII) equation

$$
\frac{d^{2} p}{d t^{2}}=\frac{1}{p}\left(\frac{d p}{d t}\right)^{2}-\frac{1}{t} \frac{d p}{d t}-\frac{1}{t}\left(p^{2}-1\right)+p^{3}-\frac{1}{p}
$$

which is closely related to studying Bonnet surfaces $[2,3]$, and the mean curvature and the metric in terms of $p(t)$ (see (3.115) in [2]). If we set $w(t)=-p(t)$, then $w(t)$ satisfies another special PIII

$$
\frac{d^{2} w}{d t^{2}}=\frac{1}{w}\left(\frac{d w}{d t}\right)^{2}-\frac{1}{t} \frac{d w}{d t}+\frac{1}{t}\left(w^{2}-1\right)+w^{3}-\frac{1}{w} .
$$

We mention that this special PIII can be expressed algebraically in terms of a fifth Painlevé transcendent and its first derivative. Consider the following pair of equations:

$$
h(\tau)=\frac{w^{\prime}(t)-w^{2}(t)-1}{w^{\prime}(t)-w^{2}(t)+1}, \quad w(t)=\frac{2 \tau h(\tau)}{\tau h^{\prime}(\tau)-h(\tau)+1},
$$

where $\tau=\frac{t^{2}}{2}$. Eliminating $h$ from (8), we get equation (7) for $w(t)$; and eliminating $w$ from (8), we get for $h(\tau)$ the special PV equation

$$
\frac{d^{2} h}{d \tau^{2}}=\left(\frac{1}{2 h}+\frac{1}{h-1}\right)\left(\frac{d h}{d \tau}\right)^{2}-\frac{1}{\tau} \frac{d h}{d \tau}-\frac{1}{8} \frac{(h-1)^{2}}{\tau^{2} h}-\frac{h}{\tau}
$$

which admits the Lax representation [15].

With the help of the preceding derivation, we can now see that equation (2) is equivalent to equation (9) after the transform

$$
h(\tau)=1+\frac{2 \sin ^{2} \frac{\Phi(x)}{2}}{\Phi^{\prime}(x)-1}, \quad \tau=-\frac{x^{2}}{8} .
$$

Hence, the connection problem of equation (2) can be studied by studying the corresponding connection problem of equation PV (9) using the isomonodromic deformation technique [7]. Based on the special Lax pair of (9), the author of [15] studied analytically this solution to equation (2) with the initial condition (3) for all real-valued $a$ and obtained the asymptotic expansion of $\Phi$ as $x \rightarrow \infty$ and explicit connection formulas by virtue of the isomonodromic deformation technique. Let us summarize the main result of [15] in the theorem as follows.

Theorem 1 There exists a unique solution of (2) which satisfies (3) for any given real number $a$. 
(A) If $a>\frac{1}{\pi}$, this solution exists for positive real $x$, and

$$
\Phi(x, a)=-x+\beta \ln x+\gamma+o(1), \quad \text { as } x \rightarrow \infty,
$$

where $\beta$ and $\gamma$ are real constants. Furthermore, the relationship between the parameters $\beta, \gamma$ in (11) and the parameter a in (3) is provided by the connection formulas

$$
\begin{aligned}
& \beta=-\frac{1}{\pi} \ln (a \pi-1), \\
& \gamma=\frac{\pi}{2}+2 \arg \Gamma\left(\frac{i \beta}{2}-\frac{1}{2}\right)+\beta \ln 2+k \pi, \quad k \in \mathbb{Z} .
\end{aligned}
$$

(B) If $a<\frac{1}{\pi}$, this solution exists for all real $x$ and increases monotonically as $x \rightarrow \infty$,

$$
\Phi(x, a)=x+\beta \ln x+\gamma+o(1)
$$

where $\beta$ and $\gamma$ are real constants. Furthermore, the relationship between the parameters $\beta, \gamma$ in (14) and the parameter a in (3) is provided by the connection formulas

$$
\begin{aligned}
& \beta=\frac{1}{\pi} \ln (1-a \pi), \\
& \gamma=-2 \arg \Gamma\left(\frac{i \beta}{2}\right)+\beta \ln 2-\pi \operatorname{sign} \beta,
\end{aligned}
$$

where $\beta \neq 0$ and $\gamma(0)=0$.

(C) If $a=\frac{1}{\pi}$, this solution exists for all real $x$ as $x$ increases monotonically to a finite limit, and, as $x \rightarrow \infty$,

$$
\Phi\left(x, \frac{1}{\pi}\right)=\frac{\pi}{2}+o(1) .
$$

In the earlier work of the authors of [4], they studied numerically this solution with the given asymptotic behavior at the origin (3) for the case of $a>\frac{1}{\pi}$ and proposed (11) and (12); however, they did not obtain the explicit expression (13) for $\gamma$. Recently, the connection formulas in Theorem 1 have been applied for calculations of the Fredholm determinant of the sine kernel $\sin \pi(x-y) / \pi(x-y)$ on the finite interval $(t,-t)$; see [11].

In this paper, we provide a simpler and more rigorous proof of Theorem 1 by using the uniform asymptotics method proposed in [1]. For our purposes, we first briefly outline some important properties of the theory of monodromy preserving deformations for the PV transcendents. The reader is referred to $[6,8]$ for more details.

One of the Lax pairs for the fifth Painlevé equation (9) is the system of linear ordinary equations [11]

$$
\begin{aligned}
& \frac{\partial \Psi}{\partial \lambda}=\left\{-i \tau \sigma_{3}+\frac{1}{\lambda}\left(\begin{array}{cc}
\frac{1}{4} & u \\
v & -\frac{1}{4}
\end{array}\right)+\frac{1}{\lambda^{2}}\left(\begin{array}{cc}
z & q \\
q & -z
\end{array}\right)\right\} \Psi, \\
& \frac{\partial \Psi}{\partial \tau}=\left\{-i \lambda \sigma_{3}+\left(\begin{array}{cc}
g & \frac{u}{\tau} \\
\frac{v}{\tau} & -g
\end{array}\right)\right\} \Psi,
\end{aligned}
$$


where $\tau=-\frac{x^{2}}{8}$ and

$$
\begin{aligned}
& z=-\frac{i}{8} \frac{h+1}{h-1}, \quad q=-\frac{1}{4} \frac{\sqrt{h}}{h-1}, \quad u+v=-\frac{i}{2 \sqrt{h}}, \\
& u-v=\frac{i \tau h_{\tau}}{(1-h) \sqrt{h}}, \quad g=\frac{1}{8 \tau}\left(1+\frac{1}{h}\right) .
\end{aligned}
$$

The compatibility condition $\Psi_{\lambda \tau}=\Psi_{\tau \lambda}$ implies that $h(\tau)$ satisfies the PV equation (9).

Equation (18) has two irregular points $\lambda=0$ and $\lambda=\infty$. There exists a canonical solution $\Psi^{(\infty)}$ defined in a neighborhood of the irregular singular point $\lambda=\infty$ with the following asymptotics behavior:

$$
\Psi^{(\infty)}(\lambda)=E^{(\infty)}(\tau)\left(I+O\left(\frac{1}{\lambda}\right)\right) \lambda^{\frac{1}{4} \sigma_{3}} \exp \left(-i \tau \lambda \sigma_{3}\right), \quad \lambda \rightarrow \infty, \arg \lambda=0
$$

where

$$
E^{(\infty)}(\tau)=\tau^{\frac{1}{8} \sigma_{3}} \exp \left(\sigma_{3} J(\tau)\right):=d^{\sigma_{3}}
$$

with $J(\tau)=\frac{1}{8} \int_{-c}^{\tau} \frac{d t}{t h(t)}$, here $c$ is a positive constant. From (18) and (21) it follows that

$$
\Psi^{(\infty)}(\lambda)=E^{(\infty)}(\tau)\left(\begin{array}{cc}
1+O\left(\frac{1}{\lambda}\right) & \frac{a_{1}}{\lambda} \\
\frac{a_{2}}{\lambda} & 1+O\left(\frac{1}{\lambda}\right)
\end{array}\right) \lambda^{\frac{1}{4} \sigma_{3}} \exp \left(-i \tau \lambda \sigma_{3}\right)
$$

with $a_{1}=\frac{u}{2 i \tau d}$ and $a_{2}=-\frac{v}{2 i \tau} d$.

On the other hand, (18) has another canonical solution $\Psi^{(0)}$ in a neighborhood of the irregular singular point $\lambda=0$

$$
\Psi^{(0)}(\lambda)=H(\tau) E^{(0)}(\tau)(I+O(\lambda)) \lambda^{\frac{1}{4} \sigma_{3}} \exp \left(\frac{i}{\lambda} \sigma_{3}\right), \quad \lambda \rightarrow 0, \arg \lambda=0,
$$

where the coefficients $H$ and $E^{(0)}$ have the form

$$
H(\tau)=\frac{1}{\sqrt{h(\tau)-1}}\left(i \sigma_{3} \sqrt{h}+\sigma_{1}\right), \quad E^{(0)}(\tau)=\tau^{\frac{1}{8} \sigma_{3}} \exp \left(-\sigma_{3} J(\tau)\right)=\tilde{d}^{\sigma_{3}} .
$$

Since $\Psi^{(\infty)}$ and $\Psi^{(0)}$ are both fundamental solutions, the connection matrix $Q$ can be defined by

$$
\Psi^{(\infty)}(\lambda)=\Psi^{(0)}(\lambda) Q
$$

Differentiating both sides of equation (26) with respect to $x$ and making use of the fact that both $\Psi^{(\infty)}$ and $\Psi^{(0)}$ satisfy (18), the isomonodromic condition $\frac{d Q}{d x}=0$ is easily found; i.e., $Q$ is a constant matrix.

In the framework of the isomonodromic deformation method, one needs to calculate the monodromy data $Q$ both in terms of the initial condition (3) and asymptotics (14). Equating then the leading terms of nontrivial monodromy data, one gets connection formulas for the parameters $\beta, \gamma$, and $a$. In the limit $x \rightarrow 0$, the first term of equation in $\lambda$ 
(18) vanishes, so the $\Psi$-functions can be expressed via the Whittaker functions [15], then the monodromy data as $x \rightarrow 0$ is calculated explicitly by use of multiplication formulas of the Whittaker functions, and we obtain that

$$
(Q)_{21}=i 2^{-3 / 4} \sqrt{a \pi} \text {. }
$$

To estimate the connection matrix in the limit $\tau \rightarrow+\infty$, one finds the WKB solution of the $\Psi$-function, which demands a standard procedure of matching near the turning points, involving parabolic cylinder functions (see [15], p. 251). Eventually, one can obtain the connection matrix for large $x$ as follows by using the asymptotics behavior of parabolic cylinder functions.

In this paper, we shall provide a hopefully simpler and more rigorous derivation of the asymptotic behavior and the connection formulas in Theorem 1 by using the uniform asymptotics method presented in [1]. Along the same lines we may find the work of Olver [12] and Dunster [5] for coalescing turning points. Initially in [1], the second Painlevé (PII) equation has been taken as an example to illustrate the method. While the difficulty in extending the techniques for PII to other transcendents is also acknowledged by the authors of [1], p. 244, yet the method has been applied to the connection problems by Wong and Zhang [17, 18], Zeng and Zhao [19], Long, Zeng, and Zhou [10]. Recently, Long et al. [9] presented a detailed asymptotics analysis of the real solutions of the first Painlevé (PI) equation by virtue of the uniform asymptotics method.

The rest of the paper is organized as follows. The proof of Theorem 1 is provided in Sect. 2. In Sect. 3, for the case of $a>\frac{1}{\pi}$ and $a<\frac{1}{\pi}$, we derive uniform approximations to the solutions of the second-order differential equation obtained from the Lax pair (18) as $x \rightarrow+\infty$ by virtue of the parabolic cylinder functions on the Stokes curves, respectively. The entry $(2,1)$ of the connection matrix $Q$ for large $x$ is also computed in the section.

\section{Proof of Theorem 1}

To prove Theorem 1, we need two lemmas.

Lemma 1 For $a>\frac{1}{\pi}$, the asymptotics behavior of the entry $(2,1)$ of the connection matrix $Q$ is

$$
(Q)_{21}=\frac{2^{-\frac{1}{4}} \sqrt{\pi} \mathrm{e}^{-\frac{\pi \beta}{4}}}{\Gamma\left(\frac{1}{2}-i \frac{\beta}{2}\right)} \exp \left(i S+\frac{i}{2} x-\frac{i \beta}{2} \ln x-\frac{i \beta}{2} \ln 2+\frac{3 \pi i}{4}\right) \text {. }
$$

Lemma 2 For $a<\frac{1}{\pi}$, the asymptotics behavior of the entry $(2,1)$ of the connection matrix $Q$ is

$$
(Q)_{21}=\frac{i \sqrt{\beta} 2^{-\frac{3}{4}} \sqrt{\pi} \mathrm{e}^{\frac{\pi \beta}{4}}}{\Gamma\left(\frac{i \beta}{2}+1\right)} \exp \left(-i S+\frac{i}{2} x+\frac{i \beta}{2} \ln x+\frac{i \beta}{2} \ln 2\right) .
$$

The rigorous proofs of those results will be given in the next section. With the help of the preceding two lemmas, we can now prove Theorem 1.

Proof of Theorem 1 We first give the proof when $a>\frac{1}{\pi}$. Since the connection matrix $Q$ must be independent of $x$, it follows that the right-hand sides of (27) and (28) are equal. 
Separating the real and imaginary parts, in view of the standard formulas $\left|\Gamma\left(\frac{1}{2}+i y\right)\right|^{2}=$ $\frac{\pi}{\cosh y \pi}$ (see [13]), gives the asymptotic behaviors (11) and connection formulas (12), (13), which completes the proof of statement $(\mathrm{A})$ in Theorem 1.

When $a<\frac{1}{\pi}$, the asymptotic behaviors (14) and connection formulas (15), (16) are obtained straightforward by equating the expressions (27) and (29); here it has been made of the formulas $\Gamma(z+1)=z \Gamma(z)$ and $|\Gamma(i y)|^{2}=\frac{2 \pi}{y\left(\mathrm{e}^{y \pi}-\mathrm{e}^{-y \pi}\right)}$ and the fact that $\Phi(x, 0)=x$ is the solution of the initial problem (2) and (3). Hence the statement (B) in Theorem 1 is proved.

Using the fact that $\Phi(x, a)$ is a continuous function of $a$ ([15], Lemma 1, p. 253) and taking (14) into consideration, according to the definition of $L$ in (98), we obtain $\lim _{x \rightarrow \infty} \Phi\left(x, \frac{1}{\pi}\right)=\frac{\pi}{2}$, which gives the proof of statement $(C)$ in Theorem 1 .

The proof of Theorem 1 is now completed.

\section{Uniform asymptotics and proofs of the lemmas}

Make the scaling

$$
\xi=x, \quad \eta=x \lambda
$$

so that (18) becomes

$$
\frac{\partial \Psi}{\partial \eta}=\left(\begin{array}{cc}
\frac{i}{8} \xi+\frac{1}{4 \eta}+\frac{\xi}{\eta^{2}} z & \frac{u}{\eta}+\frac{\xi}{\eta^{2}} q \\
\frac{v}{\eta}+\frac{\xi}{\eta^{2}} q & -\left(\frac{i}{8} \xi+\frac{1}{4 \eta}+\frac{\xi}{\eta^{2}} z\right)
\end{array}\right) \Psi
$$

Let $\left(\Psi_{1}, \Psi_{2}\right)^{T}$ be an independent solution of (31), and set

$$
\phi=\left(\frac{v}{\eta}+\frac{\xi}{\eta^{2}} q\right)^{-\frac{1}{2}} \Psi_{2}
$$

we get from (3) the second-order linear differential equation for $\phi(\eta)$

$$
\begin{aligned}
\frac{d^{2} \phi}{d \eta^{2}}= & \left\{\xi^{2}\left(\frac{i}{8}+\frac{z}{\eta^{2}}\right)^{2}+\frac{\xi}{2 \eta}\left(\frac{i}{8}+\frac{z}{\eta^{2}}\right)+\frac{1}{16 \eta^{2}}+\xi^{2} \frac{q^{2}}{\eta^{4}}+\frac{1}{\eta^{2}}\left[u \nu+\frac{\xi}{\eta} q(u+v)\right]\right. \\
& \left.+\frac{1}{4 \eta^{2}}+\xi \frac{2 z}{\eta^{3}}-\xi \frac{1}{\eta}\left(\frac{i}{8}+\frac{z}{\eta^{2}}\right) l_{1}-\frac{1}{4 \eta^{2}} l_{1}+\frac{3}{4 \eta^{2}} l_{1}^{2}-\frac{1}{\eta^{2}} l_{2}\right\} \phi,
\end{aligned}
$$

where

$$
l_{1}=\frac{v+\frac{2 \xi}{\eta} q}{v+\frac{\xi}{\eta} q}, \quad l_{2}=\frac{v+\frac{3 \xi}{\eta} q}{v+\frac{\xi}{\eta} q}
$$

From (20) it is easy to verify that

$$
z^{2}+q^{2}=-\frac{1}{64}, \quad q(u+v)=\frac{i}{8(h-1)} .
$$

Substituting (35) into (33) yields

$$
\frac{d^{2} \phi}{d \eta^{2}}=\left\{-\frac{\xi^{2}}{64}\left(1-\frac{1}{\eta^{2}}\right)^{2}+\frac{\xi^{2}}{64 \eta^{2}}(16 i z-2)+\frac{\xi}{2 \eta}\left(\frac{i}{8}+\frac{z}{\eta^{2}}\right)-\frac{\xi}{\eta}\left(\frac{i}{8}+\frac{z}{\eta^{2}}\right) l_{1}\right.
$$




$$
\left.+\frac{u v}{\eta^{2}}+\xi \frac{2 z}{\eta^{3}}+\frac{i \xi}{8 \eta^{3}(h-1)}+\frac{1}{\eta^{2}}\left(\frac{5}{16}-\frac{1}{4} l_{1}+\frac{3}{4} l_{1}^{2}-l_{2}\right)\right\} \phi
$$

\subsection{Proof of Lemma 1}

To prove Lemma 1, we need several lemmas. First, we need to construct the uniform asymptotics solution of equation (36) as $\xi \rightarrow \infty$ for $a>\frac{1}{\pi}$. When $a>\frac{1}{\pi}$, according to [15], (A.71), the solution of boundary value problem (2)-(3) has the following asymptotic expansion:

$$
\Phi^{\prime}(\xi, a)=-1+\frac{\varphi(S)}{\xi}+O\left(\xi^{-2}\right), \quad \text { as } \xi \rightarrow \infty
$$

where $\varphi(S)=\sin 4 S+2 k^{2} \sin ^{2} 2 S$ with $S=\frac{1}{2} \Phi(\xi, a)$.

It follows from the expression of $h$ in (10) that

$$
h(\xi)=\cos ^{2} S\left(1-\frac{\varphi(S) \tan ^{2} S}{2 \xi}+O\left(\xi^{-2}\right)\right), \quad \text { as } \xi \rightarrow \infty .
$$

From (38) and (20), we obtain the following asymptotic behaviors as $\xi \rightarrow \infty$ :

$$
\begin{aligned}
& z=\frac{i}{8}\left(1+2 \cot ^{2} S-\frac{\varphi(S) \csc ^{2} S}{\xi}+O\left(\xi^{-2}\right)\right) \\
& u v=\frac{\xi^{2}}{16 \sin ^{2} S}\left(1-\frac{2 \tan S+\varphi(S)\left(1+\frac{1}{2} \tan ^{2} S\right)}{\xi}+O\left(\xi^{-2}\right)\right) \\
& \frac{q}{v}=\frac{i \cot S}{\xi}\left(1+O\left(\xi^{-1}\right)\right) \\
& q v=-\frac{i \xi \cot S}{16 \sin ^{2} S}\left(1+O\left(\xi^{-1}\right)\right) .
\end{aligned}
$$

Then, for large $\xi$, substituting (39), (40), and (41) into (36), a straightforward calculation gives

$$
\frac{d^{2} \phi}{d \eta^{2}}=-\xi^{2} F(\xi, \eta) \phi
$$

where

$$
F(\xi, \eta)=\frac{1}{64}\left(1-\frac{1}{\eta^{2}}\right)^{2}+\frac{F_{1}(\xi, \eta)}{\xi}+F_{2}(\eta) O\left(\frac{1}{\xi^{2}}\right)
$$

here

$$
F_{1}(\xi, \eta)=\frac{k^{2}}{4 \eta^{2}}+\frac{i}{8 \eta}\left(1-\frac{1}{\eta^{2}}\right)\left(\frac{1}{2}+\frac{1}{b \eta-1}\right)
$$

with $b=i \tan S$, and

$$
F_{2}(\eta)=\frac{1}{\eta^{2}}+\frac{1}{\eta^{3}}
$$


For large $\xi$, it follows from equation (43) that there are two coalescing turning points near $\eta=1$ and two close to $\eta=-1$. In the present paper, we are only concerned with the two turning points, say $\eta_{1}$ and $\eta_{2}$, near $\eta=1$. When $\eta_{j}$ approach 1 , it follows from (45) that

$$
F_{1}(\xi, 1) \sim \frac{k^{2}}{4} .
$$

By using (43) and (47), we get the asymptotic formulas for the two turning points

$$
\eta_{j}^{-1}=1 \pm 2 \xi^{-1 / 2} \sqrt{k^{2}}(1+o(1)), \quad j=1,2,
$$

which coalesce to 1 when $\xi \rightarrow \infty$, and the Stokes curves defined by

$$
\Im\left(\xi\left(\eta+\frac{1}{\eta}\right)\right)=0 .
$$

Assume that $\xi \in \mathbb{R}^{+}$, then it follows from (49) that the Stokes lines of the solution $\phi$ to (43) are the positive and the negative real lines in the $\eta$ plane.

According to the philosophy of uniform asymptotics in [1], we define a number $\alpha$ by

$$
\frac{1}{2} \pi i \alpha^{2}=\int_{-\alpha}^{\alpha}\left(\tau^{2}-\alpha^{2}\right)^{1 / 2} d \tau=\int_{\eta_{1}}^{\eta_{2}} F^{1 / 2}(\xi, s) d s,
$$

and a new variable $\zeta$ by

$$
\int_{\alpha}^{\zeta}\left(\tau^{2}-\alpha^{2}\right)^{1 / 2} d \tau=\int_{\eta_{2}}^{\eta} F^{1 / 2}(\xi, s) d s .
$$

Here and in (50), the cut for the integrand on the left-hand side is the line segment joining $-\alpha$ and $\alpha$. The path of integration is taken along the upper edge of the cut. With $\alpha$ and $\zeta$ so chosen, the following lemma is a result from [1], Theorem 1.

Lemma 3 Given any solution $\phi(\eta, \xi)$ of (43), there exist constants $c_{1}, c_{2}$ such that, uniformly for $\eta$ on the Stokes cures defined by (49), as $\xi \rightarrow+\infty$,

$$
\begin{aligned}
\phi(\eta, \xi)= & \left(\frac{\zeta^{2}-\alpha^{2}}{F(\xi, \eta)}\right)^{\frac{1}{4}}\left\{\left[c_{1}+o(1)\right] D_{\nu}\left(\mathrm{e}^{\pi i / 4} \sqrt{2 \xi} \zeta\right)\right. \\
& \left.+\left[c_{2}+o(1)\right] D_{-\nu-1}\left(\mathrm{e}^{-\pi i / 4} \sqrt{2 \xi \zeta}\right)\right\},
\end{aligned}
$$

where $D_{v}(z)$ and $D_{-v-1}(z)$ are solutions of the parabolic cylinder equation and $v$ is defined by

$$
v=-\frac{1}{2}+\frac{1}{2} i \xi \alpha^{2}
$$

The next thing to do in calculating the connection matrix $Q$ as $\xi \rightarrow+\infty$ is to clarify the relation between $\zeta$ and $\eta$ in (51). 
Lemma 4 For large $\xi$ and $\eta$,

$$
\frac{1}{2} \zeta^{2}=\frac{\alpha^{2}}{2} \ln \zeta+\frac{1}{8}\left(\eta+\frac{1}{\eta}\right)-\frac{1}{4}+\frac{i}{4 \xi} \ln \eta-\frac{i}{2 \xi} \ln \left(1-b^{-1}\right)+o\left(\xi^{-1}\right)
$$

where $b=i \tan S$, and

$$
\alpha^{2}=-\frac{k^{2}}{\xi}+o\left(\frac{1}{\xi}\right) \quad \text { as } \xi \rightarrow \infty
$$

Remark 1 Coupling (53) and (55) determines the approximate value

$$
v=-\frac{i k^{2}}{2}-\frac{1}{2}+o(1) \quad \text { as } \xi \rightarrow+\infty
$$

for the order of the parabolic cylinder function $D_{v}\left(\mathrm{e}^{\pi i / 4} \sqrt{2 \xi} \zeta\right)$ in (52).

Proof The idea to prove Lemma 4 is to compute the asymptotic behavior of the integrals on the two sides of (51). A straightforward integration on the left-hand side of (51) yields

$$
\int_{\alpha}^{\zeta}\left(\tau^{2}-\alpha^{2}\right)^{1 / 2} d \tau=\frac{1}{2}\left\{\zeta\left(\zeta^{2}-\alpha^{2}\right)^{1 / 2}-\alpha^{2} \ln \left(\zeta+\left(\zeta^{2}-\alpha^{2}\right)^{1 / 2}\right)+\alpha^{2} \ln \alpha\right\}
$$

Here, the cut for the integrand is again the line segment joining $-\alpha$ and $\alpha$, and again we take the integration path along the upper edge of the cut. Because we are going to calculate the higher-order part of the both sides of (51), we will simply ignore the lower-order part in two sides, then we obtain that from (57) for large $\zeta$

$$
\frac{1}{2} \zeta^{2}-\frac{1}{2} \alpha^{2} \ln (2 \zeta)-\frac{1}{4} \alpha^{2}+\frac{1}{2} \alpha^{2} \ln (\alpha)+O\left(\alpha^{4} \zeta^{-2}\right)=\int_{\eta_{2}}^{\eta} F^{1 / 2}(\xi, s) d s .
$$

To calculate the right-hand side of (58), we split the right-hand side into two integrals respectively:

$$
\int_{\eta_{2}}^{\eta} F^{1 / 2}(\xi, s) d s=\left(\int_{\eta_{2}}^{\eta^{*}}+\int_{\eta^{*}}^{\eta}\right) F^{1 / 2}(\xi, s) d s:=I_{1}+I_{2},
$$

where

$$
\eta^{*}=1+2 T \xi^{-1 / 2},
$$

and $T$ is a large parameter to be specified more precisely later. In $I_{1}$ we take the change $s=1+2 t \xi^{-1 / 2}$, and ignore $F_{2}$, then $I_{1}$ can be evaluated for large $\xi$ as follows:

$$
I_{1}=\frac{T^{2}}{2 \xi}+\frac{k^{2}}{4 \xi}+\frac{k^{2}}{2 \xi} \ln (2 T)-\frac{k^{2}}{4 \xi} \ln \left(-k^{2}\right)+o\left(\xi^{-1}\right) .
$$

Taking $T=-\sqrt{-k^{2}}$ in $I_{1}$, we obtain (55). 
When $|\eta| \rightarrow \infty$, it follows from (46) that $F_{2}=O\left(\eta^{-2}\right)$, thus we can ignore $F_{2}$ in the integral $I_{2}$ in (59), by the binomial expansion, then $I_{2}$ is given by

$$
I_{2} \approx \int_{\eta^{*}}^{\eta} \frac{1}{8}\left(1-\frac{1}{s^{2}}\right) d s+\frac{4}{\xi} \int_{\eta^{*}}^{\eta} \frac{F_{1}(\xi, s)}{1-\frac{1}{s^{2}}} d s
$$

with the error term $o\left(\xi^{-1}\right)$.

Since

$$
\frac{4 F_{1}(\xi, s)}{1-\frac{1}{s^{2}}}=\frac{k^{2} / 2}{s-1}-\frac{k^{2} / 2}{s+1}-\frac{i / 4}{s}+\frac{i b / 2}{b s-1},
$$

then the second term in (62) is equal to

$$
\begin{aligned}
\frac{4}{\xi} \int_{\eta^{*}}^{\eta} \frac{F_{1}(\xi, s)}{1-\frac{1}{s^{2}}} d s= & \frac{1}{\xi}\left\{\frac{i}{4} \ln \eta-\frac{k^{2}}{2} \ln \left(2 T \xi^{-\frac{1}{2}}\right)+\frac{k^{2}}{2} \ln 2-\frac{i}{2} \ln \left(1-b^{-1}\right)\right. \\
& \left.+O\left(\eta^{-1}\right)+O\left(T \xi^{-\frac{1}{2}}\right)\right\}
\end{aligned}
$$

whilst the first term in $I_{2}$ is equal to

$$
\int_{\eta^{*}}^{\eta} \frac{1}{8}\left(1-\frac{1}{s^{2}}\right) d s=\frac{1}{8}\left(\eta+\frac{1}{\eta}\right)-\frac{1}{4}-\frac{T^{2}}{2 \xi}+O\left(T^{3} \xi^{-\frac{3}{2}}\right) .
$$

Combining (59), (61), (62), (64), and (65) yields

$$
\begin{aligned}
\int_{\eta_{2}}^{\eta} F^{1 / 2}(\xi, s) d s= & \frac{1}{8}\left(\eta+\frac{1}{\eta}\right)-\frac{1}{4}+\frac{k^{2}}{4 \xi}+\frac{i}{4 \xi} \ln \eta-\frac{i}{2 \xi} \ln \left(1-b^{-1}\right)+\frac{k^{2}}{2 \xi} \ln 2 \\
& -\frac{k^{2}}{2 \xi} \ln \frac{\sqrt{-k^{2}}}{\xi^{1 / 2}}+O\left(T^{3} \xi^{-\frac{3}{2}}\right)+O\left(T \xi^{-2}\right)+o\left(\xi^{-1}\right)
\end{aligned}
$$

and so, choosing $T<\xi^{\frac{1}{6}}$ and using (55) and (58), we obtain (54), which completes the proof of Lemma 4.

Lemma 5 When $\eta \rightarrow 0$, for large $\xi$, such that $\xi \eta=o(1)$, the following holds:

$$
\frac{1}{2} \zeta^{2}=\frac{\alpha^{2}}{2} \ln \zeta+\frac{1}{8}\left(\eta+\frac{1}{\eta}\right)-\frac{1}{4}-\frac{i}{4 \xi} \ln \eta-\frac{i}{2 \xi} \ln (1-b)-\frac{1}{2} \pi i \alpha^{2}+o\left(\xi^{-1}\right) .
$$

Proof The idea to prove Lemma 5 is to compute the asymptotic behavior of the integral on the right-hand side integral in (58). When $\eta \rightarrow 0$, let $\eta_{*}=1-2 T \xi^{-\frac{1}{2}}$, where $T$ is a large parameter, and split the right-hand side integral in (58) into two parts:

$$
\int_{\eta_{2}}^{\eta} F^{1 / 2}(\xi, s) d s=\left(\int_{\eta_{2}}^{\eta_{*}}+\int_{\eta_{*}}^{\eta}\right) F^{1 / 2}(\xi, s) d s=J_{1}+J_{2} .
$$

The integral $J_{1}$ can be calculated by the similar manner as in computing $I_{1}(61)$, it follows that

$$
J_{1}=\frac{T^{2}}{2 \xi}+\frac{k^{2}}{4 \xi}+\frac{k^{2}}{2 \xi} \ln (-2 T)-\frac{k^{2}}{4 \xi} \ln \left(-k^{2}\right)+O\left(\xi^{-1} T^{-2}\right)+O\left(T \xi^{-2}\right) .
$$


For the second integral $J_{2}$, according to the expressions of $F_{1}$ and $F_{2}$ in (45) and (46), respectively, we have $F_{1} \sim \eta^{-3}, F_{2}(\xi, \eta) \sim \eta^{-3}$ as $\eta \rightarrow 0$. Thus we have

$$
\begin{aligned}
J_{2}= & \int_{\eta_{*}}^{\eta}\left[\frac{1}{64}\left(1-\frac{1}{s^{2}}\right)^{2}+\frac{1}{\xi} F_{1}(\xi, s)+s^{-3} O\left(\xi^{-2}\right)\right]^{\frac{1}{2}} d s \\
= & \int_{\eta_{*}}^{\eta} \frac{1}{8}\left(1-\frac{1}{s^{2}}\right) d s+\frac{4}{\xi} \int_{\eta_{*}}^{\eta} \frac{F_{1}(\xi, s)}{1-\frac{1}{s^{2}}} d s+O\left(\xi^{-2} \ln \eta\right)+O\left(\xi^{-1} T^{-2}\right)+O\left(\xi^{-3 / 2} T^{-1}\right) \\
= & \frac{1}{8}\left(\eta+\frac{1}{\eta}\right)-\frac{1}{4}-\frac{i}{4 \xi} \ln \eta-\frac{i}{2 \xi} \ln (1-b)+\frac{k^{2}}{2 \xi} \ln 2-\frac{k^{2}}{2 \xi} \ln \left(2 T \xi^{1 / 2}\right) \\
& -\frac{T^{2}}{2 \xi}+O\left(T^{3} \xi^{-\frac{3}{2}}\right)+O\left(T \xi^{-2}\right)+o\left(\xi^{-1}\right) .
\end{aligned}
$$

Combining (68), (69), and (70) and choosing $T<\xi^{\frac{1}{6}}$, we have

$$
\begin{aligned}
\int_{\eta_{2}}^{\eta} F^{1 / 2}(\xi, s) d s= & \frac{1}{8}\left(\eta+\frac{1}{\eta}\right)-\frac{1}{4}+\frac{k^{2}}{4 \xi}-\frac{i}{4 \xi} \ln \eta-\frac{i}{2 \xi} \ln (1-b)+\frac{k^{2}}{2 \xi} \ln 2 \\
& -\frac{1}{2} \pi i \alpha^{2}-\frac{k^{2}}{4 \xi} \ln \frac{-k^{2}}{\xi}+o\left(\xi^{-1}\right) .
\end{aligned}
$$

Substituting this into (58) yields (67). The proof is completed.

We are now turning to the proof of Lemma 1.

Proof of Lemma 1 We will concentrate on evaluating the connection matrix $Q$, for which we need the uniform asymptotic behaviors of $\phi$. From the definition of $Q$ (26) it follows that

$$
\Psi_{21}^{(\infty)}=(Q)_{11} \Psi_{21}^{(0)}+(Q)_{21} \Psi_{22}^{(0)} .
$$

Hence, the first task is to find the expressions of $\Psi_{21}^{(\infty)}, \Psi_{21}^{(0)}$, and $\Psi_{22}^{(0)}$, respectively. For large $\xi$, it follows from Lemma 3 that two linearly independent asymptotic solutions of equation (43) are $\tilde{\phi}_{v}$ and $\tilde{\phi}_{-v-1}$ which are uniform with respect to $\eta$ on the Stokes curves. Here

$$
\tilde{\phi}_{\nu}=\left(\frac{\zeta^{2}-\alpha^{2}}{F(\xi, \eta)}\right)^{\frac{1}{4}} D_{\nu}\left(\mathrm{e}^{\pi i / 4} \sqrt{2 \xi \zeta}\right)
$$

and

$$
\tilde{\phi}_{-\nu-1}=\left(\frac{\zeta^{2}-\alpha^{2}}{F(\xi, \eta)}\right)^{\frac{1}{4}} D_{-\nu-1}\left(\mathrm{e}^{-\pi i / 4} \sqrt{2 \xi} \zeta\right) .
$$

By virtue of (32), we have

$$
\Psi_{21}^{(\infty)}=\left(\frac{v}{\eta}+\frac{\xi q}{\eta^{2}}\right)^{1 / 2}\left(f_{1} \tilde{\phi}_{v}+f_{2} \tilde{\phi}_{-v-1}\right)
$$

where $f_{j}(j=1,2)$ are undetermined constants which can be determined by $(23)$. 
Similarly, we obtain

$$
\begin{aligned}
& \Psi_{21}^{(0)}=\left(\frac{v}{\eta}+\frac{\xi q}{\eta^{2}}\right)^{1 / 2}\left(\delta_{1} \tilde{\phi}_{v}+\delta_{2} \tilde{\phi}_{-v-1}\right), \\
& \Psi_{22}^{(0)}=\left(\frac{v}{\eta}+\frac{\xi q}{\eta^{2}}\right)^{1 / 2}\left(\delta_{3} \tilde{\phi}_{v}+\delta_{4} \tilde{\phi}_{-v-1}\right),
\end{aligned}
$$

where $\delta_{j}(j=1,2,3,4)$ are undetermined constants which can be determined by (24).

Substituting (75), (76), and (77) into (72), then comparing with the coefficients of $\tilde{\phi}_{v}$ and $\tilde{\phi}_{-v-1}$, respectively, we get

$$
\left\{\begin{array}{l}
\delta_{1}(Q)_{11}+\delta_{3}(Q)_{21}=f_{1}, \\
\delta_{2}(Q)_{11}+\delta_{4}(Q)_{21}=f_{2},
\end{array}\right.
$$

which gives us that

$$
(Q)_{21}=\frac{\delta_{1} f_{2}-\delta_{2} f_{1}}{\delta_{1} \delta_{4}-\delta_{2} \delta_{3}}
$$

To calculate $f_{1}, f_{2}$, and $\delta_{j}(j=1, \ldots, 4)$, we proceed as follows. We shall be interested in finding the asymptotic behavior of $\tilde{\phi}_{v}$ and $\tilde{\phi}_{-v-1}$ in (73) (74) for $\eta$ on the Stokes line $\arg \eta=0$ as $\eta \rightarrow \infty$ and $\eta \rightarrow 0$, respectively. Then, substituting the obtained results into (75), (76), and (77), we will obtain the asymptotic behavior of $\Psi_{21}^{(\infty)}, \Psi_{21}^{(0)}$, and $\Psi_{22}^{(0)}$, respectively, which contain the constants $f_{1}, f_{2}$, and $\delta_{j}(j=1, \ldots, 4)$. Combining with the boundary conditions (23) and (24) for $\Psi^{(\infty)}$ and $\Psi^{(0)}$, one can determine the constants $f_{1}, f_{2}$, and $\delta_{j}(j=1, \ldots, 4)$. From [13], we have the asymptotic behavior of $D_{v}(z)$ for $|z| \rightarrow \infty$ as follows:

$$
D_{v}(z) \sim \begin{cases}z^{v} \mathrm{e}^{-\frac{1}{4} z^{2}}, & \arg z \in\left(-\frac{3}{4} \pi, \frac{3}{4} \pi\right), \\ z^{v} \mathrm{e}^{-\frac{1}{4} z^{2}}-\frac{\sqrt{2 \pi}}{\Gamma(-v)} \mathrm{e}^{i \pi v} z^{-v-1} \mathrm{e}^{\frac{1}{4} z^{2}}, & \arg z=\frac{3}{4} \pi, \\ \mathrm{e}^{-2 \pi i v} z^{v} \mathrm{e}^{-\frac{1}{4} z^{2}}-\frac{\sqrt{2 \pi}}{\Gamma(-\nu)} \mathrm{e}^{i \pi v} z^{-v-1} \mathrm{e}^{\frac{1}{4} z^{2}}, & \arg z=\frac{5}{4} \pi .\end{cases}
$$

For $\eta$ on the Stokes line $\arg \eta=0$ and $\eta \rightarrow \infty$, from (54) it immediately follows that $\zeta^{2} \sim$ $\frac{1}{4} \eta$, then we have $\arg \zeta \sim 0$. Therefore, $\arg \left(\mathrm{e}^{\pi i / 4} \sqrt{2 \xi} \zeta\right) \sim \frac{\pi}{4}$ and $\arg \left(\mathrm{e}^{-\pi i / 4} \sqrt{2 \xi} \zeta\right) \sim-\frac{\pi}{4}$ for $\xi>0$. From (43) we have $F^{-1 / 4} \sim 2^{\frac{3}{2}}$ as $\eta \rightarrow \infty$ for large $\xi$. Since $\left(\zeta^{2}-\alpha^{2}\right)^{1 / 4} \sim \zeta^{1 / 2}$ as $\eta \rightarrow \infty$, by using the appropriate asymptotic formulas of $D_{v}(z)$ in (79), we obtain from (73) and (54) that

$$
\tilde{\phi}_{\nu} \sim A_{0} \eta^{\frac{1}{4}} \mathrm{e}^{-\frac{1}{8} i \xi \eta}, \quad \text { as } \eta \rightarrow \infty,
$$

from (53), we have

$$
A_{0}=2^{\frac{v+3}{2}} \mathrm{e}^{\frac{\pi i}{4} v} \mathrm{e}^{\frac{1}{4} \xi+\frac{v}{2} \ln \xi}\left(1-b^{-1}\right)^{-\frac{1}{2}} .
$$

Similarly, from (74) and (54) it follows that

$$
\tilde{\phi}_{-\nu-1} \sim B_{0} \eta^{-\frac{1}{4}} \mathbf{e}^{\frac{1}{8} i \xi \eta}, \quad \text { as } \eta \rightarrow \infty,
$$


where

$$
B_{0}=2^{1-\frac{v}{2}} \mathrm{e}^{\frac{\pi i}{4}(v+1)} \mathrm{e}^{-\frac{i}{4} \xi-\frac{v+1}{2} \ln \xi}\left(1-b^{-1}\right)^{\frac{1}{2}} .
$$

Substituting (80) and (82) into (75) yields

$$
\Psi_{21}^{(\infty)}(\eta) \sim v^{\frac{1}{2}} \eta^{-\frac{1}{2}}\left(f_{1} A_{0} \eta^{\frac{1}{4}} \mathrm{e}^{-\frac{1}{8} i \xi \eta}+f_{2} B_{0} \eta^{-\frac{1}{4}} \mathrm{e}^{\frac{1}{8} i \xi \eta}\right) \quad \text { as } \eta \rightarrow \infty .
$$

Moreover, it has the asymptotic behavior prescribed in (23) when $\eta \rightarrow \infty$. Thus, we have

$$
v^{\frac{1}{2}} \eta^{-\frac{1}{2}}\left(f_{1} A_{0} \eta^{\frac{1}{4}} \mathrm{e}^{-\frac{1}{8} i \xi \eta}+f_{2} B_{0} \eta^{-\frac{1}{4}} \mathrm{e}^{\frac{1}{8} i \xi \eta}\right) \sim-\frac{d}{2 i \tau} \nu \lambda^{-\frac{3}{4}} \exp (-i \tau \lambda) .
$$

Comparing the coefficients of $\mathrm{e}^{\frac{1}{8} i \xi \eta}$ and $\mathrm{e}^{-\frac{1}{8} i \xi \eta}$ on both sides of the above asymptotic equation, we get

$$
f_{1}=0, \quad f_{2}=-i 2^{2} \xi^{-\frac{5}{4}} d v^{\frac{1}{2}} B_{0}^{-1} .
$$

For $\eta$ on the Stokes line $\arg \eta=0$ and $\eta \rightarrow 0$, from (67) it immediately follows that $\zeta^{2} \sim \frac{1}{4 \eta}$, then we have $\arg \zeta \sim \pi$. Therefore, $\arg \left(\mathrm{e}^{\pi i / 4} \sqrt{2 \xi} \zeta\right) \sim \frac{5 \pi}{4}$ and $\arg \left(\mathrm{e}^{-\pi i / 4} \sqrt{2 \xi} \zeta\right) \sim \frac{3 \pi}{4}$ for $\xi>0$. From (43) we have $F^{-1 / 4} \sim 2^{\frac{3}{2}} \eta$ as $\eta \rightarrow 0$ for large $\xi$. Since $\left(\zeta^{2}-\alpha^{2}\right)^{1 / 4} \sim \zeta^{1 / 2}$ as $\eta \rightarrow 0$, by using the appropriate asymptotic formulas of $D_{v}(z)$ in (79), we obtain from (73) and (67) that

$$
\tilde{\phi}_{v} \sim \eta\left(C_{0} \eta^{-\frac{1}{4}} \mathrm{e}^{-\frac{i}{8 \eta} \xi}-\frac{\sqrt{2 \pi}}{\Gamma(-v)} \mathrm{e}^{i \pi v} D_{0} \eta^{\frac{1}{4}} \mathrm{e}^{\frac{i}{8 \eta} \xi}\right), \quad \text { as } \eta \rightarrow 0,
$$

where

$$
\begin{aligned}
& C_{0}=2^{\frac{\nu+3}{2}} \exp \left(\frac{5 \pi i}{4} v+\frac{1}{2} \pi i\right) \exp \left(\frac{i}{4} \xi+\frac{v}{2} \ln \xi\right)(1-b)^{-\frac{1}{2}} \\
& D_{0}=2^{1-\frac{v}{2}} \exp \left(-\frac{5 \pi i}{4} v-\frac{3}{4} \pi i\right) \exp \left(-\frac{i}{4} \xi-\frac{v+1}{2} \ln \xi\right)(1-b)^{\frac{1}{2}} .
\end{aligned}
$$

Similarly, from (74) and (67) it follows that

$$
\tilde{\phi}_{-\nu-1} \sim \eta\left(D_{0} \mathrm{e}^{\frac{\pi i}{2}(v+1)} \eta^{\frac{1}{4}} \mathrm{e}^{\frac{i}{8 \eta} \xi}-\frac{\sqrt{2 \pi}}{\Gamma(v+1)} \mathrm{e}^{-\frac{3 \pi i}{2} \nu-\pi i} C_{0} \eta^{-\frac{1}{4}} \mathrm{e}^{-\frac{i}{8 \eta} \xi}\right), \quad \text { as } \eta \rightarrow 0 .
$$

Substituting (87) and (90) into (76) and (77), respectively, we obtain

$$
\begin{aligned}
& \Psi_{21}^{(0)} \sim \xi^{\frac{1}{2}} q^{\frac{1}{2}}\left[\left(\delta_{1}-\delta_{2} \frac{\sqrt{2 \pi}}{\Gamma(v+1)} \mathrm{e}^{-\frac{3 \pi i}{2} \nu-\pi i}\right) C_{0} \eta^{-\frac{1}{4}} \mathrm{e}^{-\frac{i}{8 \eta} \xi}\right. \\
&\left.+\left(\delta_{2} \mathrm{e}^{\frac{\pi i}{2}(v+1)}-\delta_{1} \frac{\sqrt{2 \pi}}{\Gamma(-\nu)} \mathrm{e}^{\pi i v}\right) D_{0} \eta^{\frac{1}{4}} \mathrm{e}^{\frac{i}{8 \eta} \xi}\right], \quad \text { as } \eta \rightarrow 0, \\
& \Psi_{22}^{(0)} \sim \xi^{\frac{1}{2}} q^{\frac{1}{2}}\left[\left(\delta_{3}-\delta_{4} \frac{\sqrt{2 \pi}}{\Gamma(v+1)} \mathrm{e}^{-\frac{3 \pi i}{2} \nu-\pi i}\right) C_{0} \eta^{-\frac{1}{4}} \mathrm{e}^{-\frac{i}{8 \eta} \xi}\right.
\end{aligned}
$$




$$
\left.+\left(\delta_{4} \mathrm{e}^{\frac{\pi i}{2}(v+1)}-\delta_{3} \frac{\sqrt{2 \pi}}{\Gamma(-v)} \mathrm{e}^{\pi i v}\right) D_{0} \eta^{\frac{1}{4}} \mathrm{e}^{\frac{i}{8 \eta} \xi}\right], \quad \text { as } \eta \rightarrow 0 .
$$

By using the boundary condition (24), we have

$$
\begin{aligned}
& \frac{\tilde{d}}{\sqrt{h-1}} \lambda^{\frac{1}{4}} \mathrm{e}^{\frac{i}{8 \lambda}} \sim \xi^{\frac{1}{2}} q^{\frac{1}{2}}\left[\left(\delta_{1}-\delta_{2} \frac{\sqrt{2 \pi}}{\Gamma(\nu+1)} \mathrm{e}^{-\frac{3 \pi i}{2} \nu-\pi i}\right) C_{0} \eta^{-\frac{1}{4}} \mathrm{e}^{-\frac{i}{8 \eta} \xi}\right. \\
& \left.+\left(\delta_{2} \mathrm{e}^{\frac{\pi i}{2}(v+1)}-\delta_{1} \frac{\sqrt{2 \pi}}{\Gamma(-\nu)} \mathrm{e}^{\pi i v}\right) D_{0} \eta^{\frac{1}{4}} \mathrm{e}^{\frac{i}{8 \eta} \xi}\right] \\
& \frac{-i \sqrt{h}}{\tilde{d} \sqrt{h-1}} \lambda^{-\frac{1}{4}} \mathrm{e}^{-\frac{i}{8 \lambda}} \sim \xi^{\frac{1}{2}} q^{\frac{1}{2}}\left[\left(\delta_{3}-\delta_{4} \frac{\sqrt{2 \pi}}{\Gamma(v+1)} \mathrm{e}^{-\frac{3 \pi i}{2} \nu-\pi i}\right) C_{0} \eta^{-\frac{1}{4}} \mathrm{e}^{-\frac{i}{8 \eta} \xi}\right. \\
& \left.+\left(\delta_{4} \mathrm{e}^{\frac{\pi i}{2}(v+1)}-\delta_{3} \frac{\sqrt{2 \pi}}{\Gamma(-v)} \mathrm{e}^{\pi i v}\right) D_{0} \eta^{\frac{1}{4}} \mathrm{e}^{\frac{i}{8 \eta} \xi}\right] .
\end{aligned}
$$

From (93) and (94) it follows that

$$
\frac{\delta_{2}}{\delta_{1}}=\frac{\mathrm{e}^{\frac{3 \pi i}{2} v+\pi i} \Gamma(v+1)}{\sqrt{2 \pi}}, \quad \frac{\delta_{4}}{\delta_{3}}=\frac{\sqrt{2 \pi} \mathrm{e}^{\frac{\pi i}{2}(\nu-1)}}{\Gamma(-v)}, \quad \delta_{3}=\frac{\frac{-i \sqrt{h}}{\bar{d} \sqrt{h-1}} \xi^{-\frac{1}{4}} q^{-\frac{1}{2}} C_{0}^{-1}}{1-\frac{2 \pi \mathrm{e}^{-\pi i v-\frac{3}{2} \pi i}}{\Gamma(-\nu) \Gamma(v+1)}} .
$$

Substituting (86) and (95) into (78) yields

$$
\begin{aligned}
(Q)_{21} & =-\frac{\sqrt{2 \pi} \mathrm{e}^{-\frac{3 \pi i}{2} \nu-\pi i}}{\Gamma(v+1)} 2^{2} d \tilde{d} \xi^{-1}(q v)^{\frac{1}{2}} B_{0}^{-1} C_{0} \frac{\sqrt{h-1}}{\sqrt{h}} \\
& =\frac{2^{-\frac{1}{4}} \sqrt{\pi} \mathrm{e}^{-\frac{\pi \beta}{4}}}{\Gamma\left(\frac{1}{2}-i \frac{\beta}{2}\right)} \exp \left(i S+\frac{i}{2} \xi-\frac{i \beta}{2} \ln \xi-\frac{i \beta}{2} \ln 2+\frac{3 \pi i}{4}\right)\left(1+O\left(\xi^{-1}\right)\right),
\end{aligned}
$$

where $\beta=k^{2}$. This completes the proof of Lemma 1 .

\subsection{Proof of Lemma 2}

For the case of $a<\frac{1}{\pi}$, we apply the result [15] (A.44)

$$
\Phi^{\prime}(\xi, a)=1+\frac{2 r^{2}}{\xi} \sin ^{2}(2 S)+O\left(\xi^{-2}\right), \quad \text { as } \xi \rightarrow \infty,
$$

where $S=\frac{1}{2} \Phi(\xi, a)$ and $a \in L$, here $L$ is defined as follows:

$$
L=\left\{a \mid \Phi(x)=\Phi(x, a) \text { is increasing as } x \rightarrow \infty, \exists x>0, \Phi(x, a)>\frac{\pi}{2}\right\} .
$$

By the same argument of approximating equation (36) in the last subsection, after a careful calculation, we obtain that

$$
\frac{d^{2} \phi}{d \eta^{2}}=-\xi^{2} \tilde{F}(\xi, \eta) \phi
$$

where

$$
\tilde{F}(\xi, \eta)=\frac{1}{64}\left(1-\frac{1}{\eta^{2}}\right)^{2}+\frac{1}{\xi}\left[-\frac{1}{4 \eta^{2}}\left(r^{2}-\frac{i}{\eta}\right)+\frac{i}{8 \eta}\left(1-\frac{1}{\eta^{2}}\right)\left(\frac{1}{2}-\frac{1}{b \eta-1}\right)\right]
$$




$$
+F_{2}(\eta) O\left(\frac{1}{\xi^{2}}\right)
$$

with $b=i \tan S$, and $\tilde{F}_{2}(\eta)=1+\frac{1}{\eta^{2}}+\frac{1}{\eta^{3}}$. We note that there are two coalescing turning points near 1 and two close to -1 . Here we will only consider the two turning points, say $\hat{\eta}_{1}$ and $\hat{\eta}_{2}$, near 1 . When $\hat{\eta}_{j}$ approach to 1 , by using (43), we get the asymptotic formulas for the two turning points:

$$
\hat{\eta}_{j}^{-1}=1 \pm 2 \xi^{-1 / 2} \sqrt{r^{2}-i}(1+o(1)), \quad j=1,2,
$$

which coalesce to 1 when $\xi \rightarrow \infty$, and the Stokes curves are defined by $\Im\left(\xi\left(\eta+\frac{1}{\eta}\right)\right)=0$. Assume that $\xi \in \mathbb{R}^{+}$, then the Stokes lines of the solution $\phi$ to (99) are the positive and the negative real lines in the $\eta$ plane. Similar to (50) and (51), if we define $\hat{\alpha}$ and $\vartheta(\eta)$ by

$$
\frac{1}{2} \pi i \hat{\alpha}^{2}=\int_{-\hat{\alpha}}^{\hat{\alpha}}\left(\tau^{2}-\alpha^{2}\right)^{1 / 2} d \tau=\int_{\hat{\eta}_{1}}^{\hat{\eta}_{2}} F^{1 / 2}(\xi, s) d s
$$

and

$$
\int_{\alpha}^{\vartheta}\left(\tau^{2}-\alpha^{2}\right)^{1 / 2} d \tau=\int_{\eta_{2}}^{\eta} F^{1 / 2}(\xi, s) d s
$$

respectively, then we have the following lemma which is similar to Lemma 3.

Lemma 6 There exist constants $\hat{c}_{1}, \hat{c}_{2}$ such that

$$
\begin{aligned}
\phi(\eta, \xi)= & \left(\frac{\vartheta^{2}-\alpha^{2}}{\tilde{F}(\xi, \eta)}\right)^{\frac{1}{4}}\left\{\left[\hat{c}_{1}+o(1)\right] D_{\nu}\left(\mathrm{e}^{\pi i / 4} \sqrt{2 \xi} \vartheta\right)\right. \\
& \left.+\left[\hat{c}_{2}+o(1)\right] D_{-\nu-1}\left(\mathrm{e}^{-\pi i / 4} \sqrt{2 \xi} \vartheta\right)\right\}
\end{aligned}
$$

as $\xi \rightarrow+\infty$ uniformly for $\eta$ on the Stokes cures, where $D_{v}(z)$ and $D_{-v-1}(z)$ are solutions of the parabolic cylinder equation, and $v$ is defined by $v=-\frac{1}{2}+\frac{1}{2} i \xi \hat{\alpha}^{2}$.

Moreover, by an argument similar to the one used in Lemma 4, we obtain the asymptotic behaviors of $\vartheta(\eta)$ as $\eta \rightarrow+\infty$ and $\eta \rightarrow 0$ for large $\xi$, and we state those results as follows without proof.

Lemma 7 For large $\xi$ and $\eta$,

$$
\frac{1}{2} \vartheta^{2}=\frac{\alpha^{2}}{2} \ln \vartheta+\frac{1}{8}\left(\eta+\frac{1}{\eta}\right)-\frac{1}{4}+\frac{i}{4 \xi} \ln \eta+\frac{i}{2 \xi} \ln \left(1-b^{-1}\right)-\frac{i}{\xi} \ln 2+o\left(\xi^{-1}\right)
$$

where $b=i \tan S$, and

$$
\hat{\alpha}^{2}=\frac{r^{2}-i}{\xi}+o\left(\frac{1}{\xi}\right) \text { as } \xi \rightarrow \infty \text {. }
$$


Remark 2 By using of the definition of $v$ in Lemma 6 and (106), we get the approximate value

$$
v=\frac{i r^{2}}{2}+o(1), \quad \text { as } \xi \rightarrow+\infty
$$

for the order of the parabolic cylinder function $D_{v}\left(\mathrm{e}^{\pi i / 4} \sqrt{2 \xi \vartheta}\right)$ in (104).

Lemma 8 When $\eta \rightarrow 0$, for large $\xi$ such that $\xi \eta=o(1)$, the following holds:

$$
\begin{aligned}
\frac{1}{2} \vartheta^{2}= & \frac{\vartheta^{2}}{2} \ln \vartheta+\frac{1}{8}\left(\eta+\frac{1}{\eta}\right)-\frac{1}{4}-\frac{i}{4 \xi} \ln \eta+\frac{i}{2 \xi} \ln (1-b) \\
& -\frac{i}{\xi} \ln 2-\frac{1}{2} \pi i \alpha^{2}+o\left(\xi^{-1}\right) .
\end{aligned}
$$

The proofs of those lemmas are analogous to those in Lemma 4 and will not be included here. Now we are in a position to prove Lemma 2.

Proof of Lemma 2 Based on Lemmas 6, 7, and 8, by suitable modification to the proof of Lemma 1 , we can show that the entry $(2,1)$ of the connection matrix $Q$ as $\xi \rightarrow+\infty$ for $a<\frac{1}{\pi}$ has the asymptotic behavior

$$
(Q)_{21}=\frac{i r 2^{-\frac{3}{4}} \sqrt{\pi} \mathrm{e}^{-\frac{\pi i}{2} v}}{\Gamma(\nu+1)} \exp \left(-i S+i \frac{1}{2} \xi+v \ln \xi+v \ln 2\right)\left(1+O\left(\xi^{-1}\right)\right)
$$

Substituting (107) into (109) and denoting $\beta=r^{2}$, we obtain (29). This completes the proof of Lemma 2.

\section{Acknowledgements}

The authors are grateful to the referees for their valuable suggestions and comments for improvement of the paper.

\section{Funding}

The work was supported in part by the National Natural Science Foundation of China (No. 11571376), Natural Science Foundation of Guangdong Province (No. 2014A030313176), Science and Technology Research Project of Jiangxi

Provincial Education Department (No. GJJ180581), and Doctoral Startup Fund of Jinggangshan University (No. JZB16001).

Availability of data and materials

Not applicable.

Ethics approval and consent to participate

Not applicable.

Competing interests

The authors declare that they have no competing interests.

\section{Authors' contributions}

Each of the authors contributed to each part of this study equally, all authors read and approved the final manuscript.

\section{Publisher's Note}

Springer Nature remains neutral with regard to jurisdictional claims in published maps and institutional affiliations.

Received: 21 September 2018 Accepted: 7 April 2019 Published online: 17 April 2019

\section{References}

1. Bassom, A.P., Clarkson, P.A., Law, C.K., McLeod, J.B.: Application of uniform asymptotics to the second Painlevé transcendent. Arch. Ration. Mech. Anal. 143, 241-271 (1998) 
2. Bobenko, A., Eitner, U.: Painlevé Equations in the Differential Geometry of Surfaces. Lect. Notes Maths. Springer, Berlin (2000)

3. Bobenko, A., Eitner, U., Kitaev, A.: Surfaces with harmonic inverse mean curvature and Painlevé equations. Geom. Dedic. 68, 187-227 (1997)

4. Creamer, D.B., Thacker, E.B., Wilkinson, D.: Some exact results for the two-point functions of an integrable quantum field theory. Phys. Rev. D 23, 3081-3084 (1981)

5. Dunster, T.M.: Asymptotic solutions of second-order linear differential equations having almost coalescent turning points, with an application to the incomplete gamma function. Proc. R. Soc. Lond. Ser. A 452, 1331-1349 (1996)

6. Fokas, A.S., Its, A.R., Kapaev, A.A., Novokshenov, V.Y.: Painlevé Transcendents: The Riemann-Hilbert Approach. AMS Mathematical Surveys and Monographs, vol. 128. Am. Math. Soc., Providence (2006)

7. Its, A.R., Novokshenov, V.Y.: The Isomonodromic Deformation Method in the Theory of Painlevé Equations. Lect. Notes Maths., vol. 1191. Springer, Berlin (1986)

8. Jimbo, M.: Monodromy problem and the boundary condition for some Painlevé equations. Publ. Res. Inst. Math. Sci. 18, 1137-1161 (1982)

9. Long, W.-G., Li, Y.T., Liu, S.-Y., Zhao, Y.-Q.: Real solutions of the first Painlevé equation with large initial data. Stud. Appl. Math. 139, 505-532 (2017)

10. Long, W.-G., Zeng, Z.-Y., Zhao, Y.-Q:: A note on the connection problem of some special Painlevé $V$ functions. Complex Var. Elliptic Equ. 62, 726-737 (2017)

11. Novokshenov, V.Y.: Level spacing functions and connection formulas for Painlevé V transcendent. Physica D 152-153, 225-231 (2001)

12. Olver, F.W.J.: Second-order linear differential equations with two turning points. Philos. Trans. R. Soc. Lond. Ser. A 278, 137-174 (1975)

13. Olver, F.W.J., Lozier, D.W., Boisvert, R.F., Clark, C.W. (eds.): NIST Handbook of Mathematical Functions. Cambridge University Press, Cambridge (2010)

14. Shukla, P.: Level spacing functions and the connection problem of a fifth Painlevé transcendent. J. Phys. A, Math. Gen. 28, 3177-3195 (1995)

15. Suleimanov, B.I.: On asymptotics of regular solutions for a special kind of Painlevé V equation. Lect. Notes Math. 1193, 230-260 (1986)

16. Vaidya, H.G., Tracy, C.A.: One particle reduced density matrix of impenetrable bosons in one dimension at zero temperature. J. Math. Phys. 20, 2291-2312 (1979)

17. Wong, R., Zhang, H.Y.: On the connection formulas of the third Painlevé transcendent. Discrete Contin. Dyn. Syst., Ser. A 23, 541-560 (2009)

18. Wong, R., Zhang, H.Y:: On the connection formulas of the fourth Painlevé transcendent. Anal. Appl. 7, 419-448 (2009)

19. Zeng, Z.-Y., Zhao, Y.-Q.: Application of uniform asymptotics to the connection formulas of the fifth Painlevé equation. Appl. Anal. 95, 390-404 (2016)

\section{Submit your manuscript to a SpringerOpen ${ }^{\circ}$ journal and benefit from:}

- Convenient online submission

- Rigorous peer review

- Open access: articles freely available online

- High visibility within the field

- Retaining the copyright to your article

Submit your next manuscript at $\boldsymbol{\nabla}$ springeropen.com 